\title{
Urgences
}

\section{Presque silence}

\section{Jacques Brault, Il n'y a plus de chemin, Saint-Lambert, Éd. du} Noroît/Table rase, 1990, 69 p.

\section{Hélène Marcotte}

Numéro 31, mars 1991

Poétique de la note

URI : https://id.erudit.org/iderudit/025641ar

DOI : https://doi.org/10.7202/025641ar

Aller au sommaire du numéro

Éditeur(s)

Urgences

ISSN

0226-9554 (imprimé)

1927-3924 (numérique)

Découvrir la revue

Citer ce compte rendu

Marcotte, H. (1991). Compte rendu de [Presque silence / Jacques Brault, Il n'y a plus de chemin, Saint-Lambert, Éd. du Noroît/Table rase, 1990, 69 p.] Urgences,

(31), 122-126. https://doi.org/10.7202/025641ar d'utilisation que vous pouvez consulter en ligne.

https://apropos.erudit.org/fr/usagers/politique-dutilisation/ 


\section{deliveline}

\section{Presque silence ${ }^{1}$}

\section{Hélène Marcotte}

"Et voici bientôt le bout du dernier chemin ${ }^{2}$ annonçait le narrateur de Trois fois passera, pressentant limminence de l'arrêt, prévoyant d'ores et déjà qu'un jour, il n'y aurait plus de chemin...

Que Jacques Brault tisse de multiples liens entre ses $œ u v r e s$ ne fait nul doute aujourd'hui. D'un livre à l'autre s'organise un réseau d'images obsédantes qui s'interpellent, se complètent et se prolongent. Ainsi, d'entrée de jeu, le titre de son dernier recueil de poésie (r)appelle ceux des volumes d'essais, respectivement intitulés Chemin faisant et $L a$ poussière du chemin. Mais qui plus est, le personnage principal d'Agonie semble renaitre dans ce petit livre qui, tant par sa présentation matérielle que par son propos, évoque le mystérieux carnet gris du professeur-clochard. Encore une fois donc, l'auteur explore l'univers d'un vagabond et, surtout, exploite le thème du chemin, du parcours. Sa marotte, comme il le dit lui-même ${ }^{3}$. Mais voilà, il n'y a plus de chemin. C'est l'arrêt, le piétinement, le cul-de-sac. C'est encore l'agonie soudaine, la vie qui a mal et ne sait plus où aller, le désir laissé à lui-même. Rapidement, le titre de recueil devient le leitmotiv et la seule réalité du narrateur.

Le recueil est divisé en quatre parties non titrées. Chaque partie est précédée d'un dessin de l'auteur et de deux citations, l'une ayant trait au vagabondage, l'autre plus générale. Il est difficile toutefois de saisir le pourquoi de ces coupures puisque les textes se lisent d'un seul trait, sans rupture évidente. Le poème liminaire indique l'orientation profonde du recueil:

1 Jacques Brault, II n'y a plus de chemin, Saint-Lambert, Ed. du Noroit/Table rase, $1990,69 \mathrm{p}$.

2 Jacques Brault, Trois fois passera, Saint-Lambert, Éd. du Noroitt, 1981, 87 p.

3 Robert Mélançon, * De la poésie et de quelques circonstances. Entretien avec Jacques Brault ", Voix \& images, Montréal, vol. XII, $n^{\circ}$ 2, hiver 1987, p. 198. 
Voici qu'on siffle un petit air ancien de mal à l'âme

a-t-on idée d'avoir une âme

et qui a mal en plus

où chante sans en avoir l'air

l'ombre de qui n'est plus

Le narrateur, pauvre cloche vagabonde prénommée Charles, prend conscience qu'il n'y a plus de chemin. Et c'est à partir de ce constat que commence l'aventure/écriture. Le recueil est structuré à la manière d'un long monologue intérieur, de sorte que la poésie demeure très près de la prose. La brièveté des phrases imite la spontanéité du discours oral. Il faut dire aussi qu'une certaine appréhension à briser le silence ralentit le débit du narrateur: "Les longues phrases, ça donne soif. Et ça rend mélancolique; on a le pressentiment que ça finira mal". La création de "Personne" permet néanmoins à Charles d'avoir "au moins un semblant de moi " à qui parler et donne au soliloque l'apparence du dialogue.

La poésie d'Il n'y a plus de chemin est une poésie du quotidien, mais d'un quotidien transfiguré, rendu "admirable" par l'écriture. Brault ne cherche pas à mettre en relief la marginalité du vagabond, et encore moins son dénuement. Bien qu'il rende avec justesse le pathétique de cette vie clocharde, il suggère davantage la misère qu'il ne la peint: "la poubelle du déjeuner", "ma chaussure pleurante", etc. L'écriture est simple, rythmée et chantante, et les fréquents emprunts faits à la langue orale, tant par le vocabulaire, la syntaxe, que le style, accentuent la vraisemblance et la justesse de ton des poèmes.

\section{Une sombre vagabonde}

La solitude et l'immobilité du clochard facilitent, voire exigent la résurrection du passé: "Les épluchures d’une vie, il y en a plein derrière. Je les ravale. On se bouffe pour continuer». Le rôle de la mémoire devient alors primordial puisque le présent n'existe que par les souvenirs: «Je parle, Personne, de choses perdues. Sans ça, je ne parlerais plus». L'écoulement du temps s'inscrit à travers les nombreux paysages évoqués et, de chemin en chemin, se révèle peu à peu l'univers du vagabond. Des êtres émergent pêle-mêle: " un drôle de marsouin prénommé François, fol et voleur», "une gentille vieille dame aux iris mauves", puis le guenillou, 
Gérard-le-visité, Anna-l'illuminée, Juan-le-foudroyé... Des lieux balisent ces rencontres: rue Saint-Zotique, la Côte-desNeiges, puis tout Montréal. Mais davantage que les incidents de parcours, ce sont les sensations éprouvées à divers moments de la vie qui renaissent. Un souvenir, une sensation; un nouveau chemin se dessine, s'impose à la mémoire:

Nous boirons d'un coup de langue sur les lèvres un air pêche et lilas, quelque chose qui a la saveur d'une joue de gamine comme quand nous jouions à nous attraper sans penser à mal, sans penser à rien. Et se déroulait alors en nous un chemin odorant. Les rues du quartier avaient des tendresses d'avant les humains. C'était un peu, mon pauvre Charles, ton vert paradis.

Au fil des heures, le vagabond évoque une multitude de chemins empruntés au hasard de l'existence: "les petits, dérobés, tout croches et empêtrés", les "chemins défoncés", les « sale[s] chemin[s]», et tente de saisir la vie dans ce qu'elle a de fugitif et d'éternel, dans ce qu'elle a d'unique et de banal. À travers les bribes décousues du passé qu'il nous livre, se décèlent un étonnement perpétuel, qui tient de l'émerveillement enfantin, de même qu'une immense tendresse envers l'humble quotidien. L'isolement n'en est que plus difficilement acceptable: «Pourquoi m'avez-vous abandonné, mondes qui m'ignoriez avec une telle douceur?"

La présence de la mort, cette "sombre vagabonde", transpire dans presque tous les poèmes. Car s'il n'y a plus de chemin, il y a la mort et le long soliloque du clochard n'est en fait qu'une longue agonie. Dès lors, constate Charles, le décor environnant importe peu. Ici ou ailleurs, "ville ou campagne, buildings ou brins d'herbe, quelle différence?" "Ce lieu sans nom ni apparence où je suis, récitant des ténèbres intérieures. Ruelle hérissée de barbelures où l'âme se clochardise", conduit au néant: «je me creuse sur place; jusqu'à toi, qui n'existes pas ».

\section{Les procédés de mise à distance ${ }^{4}$}

Les procédés de mise à distance du récit utilisés dans le recueil servent à désamorcer le tragique de la situation. Fréquemment, Charles exprime son désarroi face à sa condition. Nulle colère cependant, nulle impatience. De l'amer-

4 Voir à ce sujet Micheline Cambron, Une société, un récit. Discours culturel au Québoc (1967-1976), Montréal, L'Hexagone, 1990, 204 p. 
tume çà et là, de la mélancolie un peu partout. Une grande lassitude aussi. En fait, il est difficile de cerner les sentiments intimes du personnage principal puisqu'il les extériorise en les personnifiant et en les acceptant pour compagnons: «Rester la. Avec l'angoisse, avec la solitude? Faudrait choisir. La place manque pour les deux. Je suis tellement fatigué. Ça va, restez, toutes les deux. J'ai l'habitude". Ce recours à l'allégorie permet au narrateur de prendre un certain recul par rapport à ses sentiments. Un déplacement s'opère: de la sensibilité, on passe à la décomposition intellectuelle.

En réfléchissant sur son propre discours, Charles prend davantage ses distances avec ses émotions: "La semaine dernière, à l'heure mauve, ma préférée, aussi timide qu'un matin lève-tard, bah ! tu bavardes comme les poètes-perroquets, à l'heure dite donc, $[. .$.$] ». Cette apostrophe souligne de manière$ subtile la coexistence du langage commun et du langage poétique à l'intérieur des textes. L'hétérogénéité des discours se manifeste aussi par la présence de poèmes en prose et de poèmes en vers dans le recueil. En effet, quelques poèmes brefs differrent des autres d'abord par leur ton, qui se veut davantage impersonnel, et ensuite par la disposition des vers qui, d'horizontale, devient verticale. Ainsi, malgré le mépris apparent du clochard pour le langage poétique et lutilisation du langage populaire, la poésie n'en demeure pas moins l'unique support du texte et permet à Charles de dépasser sa condition. Par la poésie, il accède à la culture savante.

À plusieurs reprises, le clochard ponctue son discours de «qu'ils disaient»: "Il paraît que l'ail, ça donne de l'affection. Qu'ils disaient", "Il paraît que je suis né sous le signe de Saturne; planète néfaste, qu'ils disaient", "L'important c'est de partir, qu'ils disaient", etc. Cette répétition marque une distanciation ironique vis-à-vis des lieux communs contenus dans le discours social. Elle exprime aussi le désenchantement de Charles qui, de façon implicite, cherche à valoriser son mode de vie en soulignant le ridicule des croyances et dictons populaires.

On a déjà signalé que, de Mémoire (1965) à Moments fragiles (1984), la poésie de Brault délaisse peu à peu la dimension collective au profit de lintime ${ }^{5}$. Toutefois, dans les

5 Gilles Marcotte, "Poésie de novembre", Voix \& images, vol. III, $n^{\circ} 2$, hiver 1987, p. 245-246. 
recueils précédant $I l$ n'y a plus de chemin, il était possible d'identifier le destinataire interne. Ici, le *tu " renvoie à Personne, le "vous" à «je ne sais qui ou quoi ", et le "nous " au couple Charles-Personne ou encore aux compagnons de jadis, c'est-à-dire à un destinataire fictif ou absent. Le besoin manifeste de compenser le manque de destinataire témoigne d'une volonté quasi désespérée d'échapper à la solitude. Cependant, même Charles ne se leurre guère sur la réalité de son interlocuteur puisqu'il entrecoupe son monologue-dialogue de réflexions comme: "De quoi, je me-te demande», "Tu ronfles, Personne. Mettons que je parle à mon chapeau ", etc.

Le sentiment de nostalgie qui domine les poèmes constitue, pour sa part, une dévalorisation du présent en regard du passé. L'idéalisation du passé trahit le désir de revenir en arrière. Conscient qu'une rupture s'est produite (il n'y a plus de chemin), Charles tente de considérer ce temps d'arrêt comme une halte et non comme un sursis. Paradoxalement, il semble conscient de cette volonté qui le pousse à fuir le réel :

On joue au juif errant. On se prend pour un autre qui se prend pour un autre qui etc. Et on s'èchappe de soi et de l'autre.

La douce imposture du "je est un autre» ne peut toutefois durer indéfiniment. À la fin du recueil, Charles doit affronter la réalité:

Donnez-moi la main. Attendez-moi. II n'y a vraiment personne? On imagine, on s'enroule dans une image, on s'invente une autre vie. Façon de mourir en douce, à petites secousses. [...] Maintenant, ça y est, je vais me mettre au trou avec la sombre vagabonde.

Les modes de mise à distance du texte que nous venons d'esquisser représentent autant de moyens pour le narrateur de nier la vie agonique. L'allégorie lui permet de se distancer de ses émotions, l'hétérogénéité des discours de dépasser sa condition, l'ironie de valoriser son discours en discréditant le discours social, la création d'un destinataire de vaincre son isolement et la nostalgie de rejeter le présent. Dans cette perspective, davantage qu'une simple rétrospective de la vie d'un clochard, ou encore qu'une réflexion générale sur l'existence, Il n'y a plus de chemin est le cri d'un homme qui se refuse à mourir. 